\title{
Chapter 56 \\ Topology Drives Calcium Wave Propagation in 3D Astrocyte Networks
}

\author{
Jules Lallouette and Hugues Berry
}

\begin{abstract}
Glial cells are non-neuronal cells that constitute the majority of cells in the human brain and significantly modulate information processing via permanent cross-talk with the neurons. Astrocytes are also themselves inter-connected as networks and communicate via chemical wave propagation. How astrocyte wave propagation depends on the local properties of the astrocyte networks is however unknown. In the present work, we investigate the influence of the characteristics of the network topology on wave propagation. Using a model of realistic astrocyte networks ( $>1000$ cells embedded in a 3D space), we show that the major classes of propagations reported experimentally can be emulated by a mere variation of the topology. Our study indicates that calcium wave propagation is favored when astrocyte connections are limited by the distance between the cells, which means that propagation is better when the mean-shortest path of the network is larger. This unusual property sheds new light on consistent reports that astrocytes in vivo tend to restrict their connections to their nearest neighbors.
\end{abstract}

\subsection{Introduction}

More than half of the cells in the human brain are glial cells. These non-neuronal cells have recently been evidenced to play a direct active role in information transfer in the brain. Indeed astrocytes (the main subtype of glial cells) not only react to but can also modulate synaptic communication between neurons [8, 16]. Astrocytes are also themselves inter-connected as networks on which chemical waves propagate [9]. Understanding astrocyte-astrocyte and astrocyte-neuron cross-talks is thus crucial to understanding the brain [8]. Chemical communication within astrocyte networks is characterized as elevations of intracellular calcium that propagate from cell to cell though protein channels called gap-junctions channels (GJC). However,

\footnotetext{
J. Lallouette $(\varangle) \cdot$ H. Berry

BEAGLE, INRIA RhôneAlpes, Université de Lyon, LIRIS, UMR5205, Villeurbanne, France e-mail: jules.lallouette@inria.fr

H. Berry

e-mail: hugues.berry@inria.fr
} 
depending on the experimental conditions, the reported speed and extent of the propagation (the number of cells that participate in the waves) vary over a large range. These discrepancies can be interpreted as many different intracellular biochemistry conditions or as different topologies of the astrocyte networks. Here, we questioned the latter hypothesis, that the various propagation ranges observed experimentally may be explained on the sole basis of the topology of astrocyte networks.

In the brain, astrocytes are believed to occupy separate spatial territories, connecting to their nearest astrocyte neighbors only [2, 15]. Beyond this general setting, the structure of astrocyte networks is still unknown. Recent experimental evidence however suggests that astrocytes networks display different topologies depending on the brain region [18] and even that neuronal activity can modify these topologies by regulating inter-astrocyte GJC [9]. In contrast, in the modeling literature, most articles consider astrocyte networks embedded in a two dimensional space [7, 13] and connected using regular lattices $[10,11,13]$. The effect of more complex or more realistic topologies has been restricted to small networks $(5-10$ cells) $[6,12]$, that do not allow comparison of propagation ranges with biological data.

In the present work, we study a model of realistic astrocyte networks ( $>1000$ cells embedded in a $3 \mathrm{~d}$ space) and investigate how the characteristics of the network topology affect calcium wave propagation at a network level. We show that indeed, the major classes of observed propagations can be emulated by a mere variation of the topology. Our study indicates that calcium wave propagation is favored when astrocyte connections are limited by the distance between the cells, which means that propagation is better when the mean-shortest path of the network is larger. Altogether, our findings offer a sound theoretical understanding of calcium wave propagations in astrocyte networks.

\subsection{The Model}

Our model consists of two main parts: a model describing calcium dynamics inside each astrocyte on the one hand, and the network topology models to connect astrocytes, on the other hand.

\subsubsection{Calcium Dynamics Model}

Albeit experimental protocols monitor wave propagation as variations of intracellular calcium, the molecule that is transmitted through GJC to connected astrocytes is not calcium but another messenger, called $\mathrm{IP}_{3}$. When $\mathrm{IP}_{3}$ level in the cell cytoplasm is large enough, a calcium surge is released from intracellular calcium stores to the cell cytoplasm. In turn, since the $\mathrm{IP}_{3}$ producing (and degrading) enzymes are activated by cytoplasmic calcium, this calcium elevation will lead to increased $\mathrm{IP}_{3}$ levels, some of which can be transported through a GJC to a connected astrocyte. 
The $\mathrm{IP}_{3}$ entering the connected cell can then regenerate the original calcium signal if the transferred $\mathrm{IP}_{3}$ amount is large enough.

To model the dynamics of this system we used the ChI model, developed and studied at the single-cell level in $[10,17]$. This model uses three coupled nonlinear equations to describe calcium dynamics in each astrocyte $i$ :

$$
\begin{aligned}
\frac{d C^{i}}{d t} & =J_{\text {chan }}\left(C^{i}, h^{i}, \mathrm{IP}_{3}^{i}\right)+J_{\text {leak }}\left(C^{i}\right)-J_{\text {pump }}\left(C^{i}\right) \\
\frac{d h^{i}}{d t} & =\left(h_{\infty}\left(C^{i}, \mathrm{IP}_{3}^{i}\right)-h^{i}\right) / \tau_{h}\left(C^{i}, \mathrm{IP}_{3}^{i}\right) \\
\frac{d \mathrm{IP}_{3}^{i}}{d t} & =P_{P L C \delta}\left(C^{i}, \mathrm{IP}_{3}^{i}\right)-D_{3 K}\left(C^{i}, \mathrm{IP}_{3}^{i}\right)-D_{5 P}\left(\mathrm{IP}_{3}^{i}\right)+J_{\text {net }}^{i}
\end{aligned}
$$

where $C^{i}, h^{i}$ and $\mathrm{IP}_{3}^{i}$ are respectively the cell-averaged calcium concentration, the fraction of open intracellular $\mathrm{IP}_{3}$ receptors at the calcium stores and the cellaveraged concentration of $\mathrm{IP}_{3}$ messenger of cell $i$. The terms in the RHS represent calcium flux between intracellular compartments or internal enzyme kinetics and can be found in $[10,17] . J_{n e t}^{i}=\sum_{j \in N(i)} J_{j \rightarrow i}$ represents the total flux of $\mathrm{IP}_{3}$ from neighbouring cells $j$ to cell $i$. To model the $\mathrm{IP}_{3}$ flux $J_{j \rightarrow i}$ though GJC between cells $j$ and $i$, we use a non-linear coupling (see [10] for justification):

$$
J_{j \rightarrow i}=\frac{F}{2}\left(1+\tanh \left(\frac{\left|\Delta_{j i} \mathrm{IP}_{3}\right|-\mathrm{IP}_{3}^{t h r}}{\mathrm{IP}_{3}^{\text {scale }}}\right)\right) \frac{\Delta_{j i} \mathrm{IP}_{3}}{\left|\Delta_{j i} \mathrm{IP}_{3}\right|}
$$

where $F$ is the coupling strength, $\Delta_{j i} \mathrm{IP}_{3}$ is the $\mathrm{IP}_{3}$ difference between cells $j$ and $i$ and $\mathrm{IP}_{3}^{t h r}$ is a threshold below which the flux is small. Note that $J_{j \rightarrow i}$ can be positive or negative, depending on the sign of the $\mathrm{IP}_{3}$ gradient.

Except when explicitly indicated below, all parameters were taken from [10] (FM encoding conditions). All the resulting coupled ODEs were numerically integrated using a 4th order Runge-Kutta scheme with a time step of $10 \mathrm{~ms}$. The model was implemented in $\mathrm{C}++$ and was run on the IN2P3 Computing Center which we thank for providing the computer resources.

\subsubsection{Network Topologies}

Spatial Structure One major goal of our study is to obtain propagation measures in the model that can be compared to experimental data (propagation extent, speed, etc.). A recent experimental study [19] has exhibited the distribution of the nearest astrocyte-astrocyte distance in mice brains (mean distance $\mu_{\text {exp }}=50 \mu \mathrm{m}$, coefficient of variation $c v_{\text {exp }}=0.25$, minimum distance $m_{\exp }=20 \mu \mathrm{m}$ ). In accordance with these results, we reproduced this distribution by first placing $N$ astrocytes at the nodes of a regular 3-dimensional cubic grid (interspacing distance $a_{\text {grid }}$ ). The position of each cell was then jittered by adding to its coordinates a random normal variable (zero mean, variance $\sigma$ ). From a simple grid search, the best match between this model and the distribution obtained in [19] was found with $a_{\text {grid }}=70 \mu \mathrm{m}$, $\sigma=55 \mu \mathrm{m}$. 

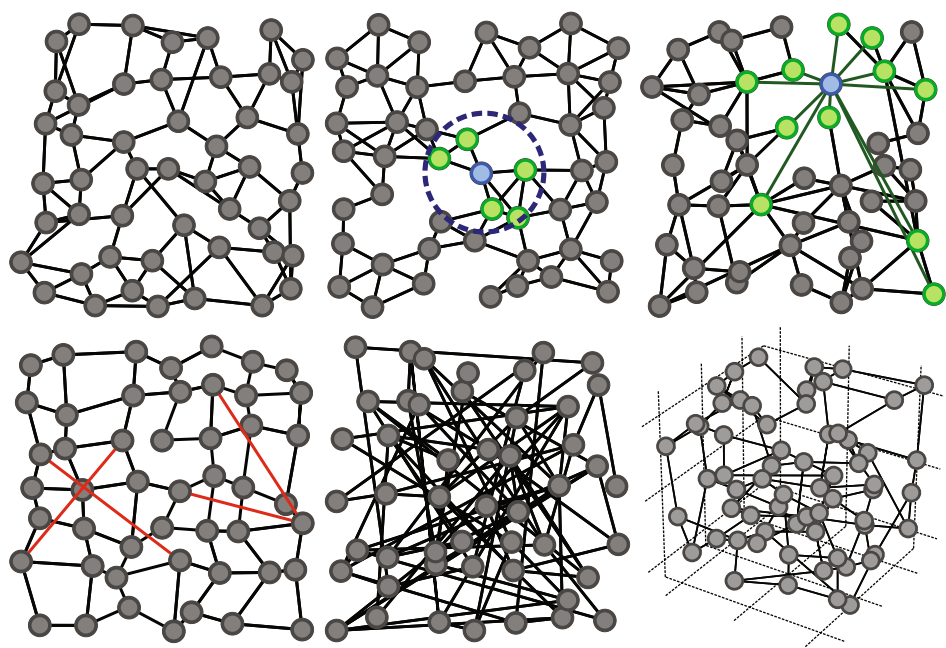

Fig. 56.1 Representations of networks obtained with each model. From left to right and top to bottom: regular degree, link radius, spatial scale free, shortcut, Erdős-Rényi and three dimensional version of a regular degree network. Note that most of these illustrations are two-dimensional for readability, but the networks used in the present study are all three-dimensional

Topological Structure We investigated five kinds of network topologies:

- Regular degree networks are constructed by linking each astrocyte to its $k_{\text {reg }}$ nearest neighbors in space.

- Link radius networks are spatial networks in which each astrocyte is linked to all the astrocytes found within distance $r_{\text {link }}$.

- Spatial scale free networks are built incrementally by the classical preferential attachment rule, but taking spatial distances into account (detailed description in [1]). In short, a parameter $\left(r_{c}\right)$ controls the trade-off between scale-free structure and the link restriction to short intercell distances.

- Shortcut networks are based on 3d cubic lattices. Each edge is then rewired with probability $p_{s}$ : one of the edge end is replaced by a (uniformly) randomly chosen cell. Those networks are similar to the classical small-world topologies.

- Erdös-Rényi networks are the only non-spatial networks: each pair of node is linked with a probability $p_{e r}$ independently of distance.

Two-dimensional illustrations of the networks created with each of these models are shown in Fig. 56.1. Each of these topologies present specific features that are not necessarily biologically realistic but will provide us insights into what kind of features networks should or should not have in order to support realistic wave propagation. In each case, we varied the parameters specific to the topology so that the mean degree of the network $2 \leq\langle k\rangle \leq 17$. For each parameter value, the measurements were averaged over 20 realizations of the topology with the given parameters. All networks had $N=1331$ astrocytes. 
Fig. 56.2 Propagation extent $N_{a c t}$, as a function of the mean degree of the networks $\langle k\rangle$. Values are grouped in classes of network models and error bars plot the standard deviation in $\log$ scale. The inset contains the propagation extent for shortcut networks with $\langle k\rangle=6$ and as a function of $p_{s}$ (red curve), and the mean propagation extent for regular degree networks, with parameter $k_{r e g}=6$

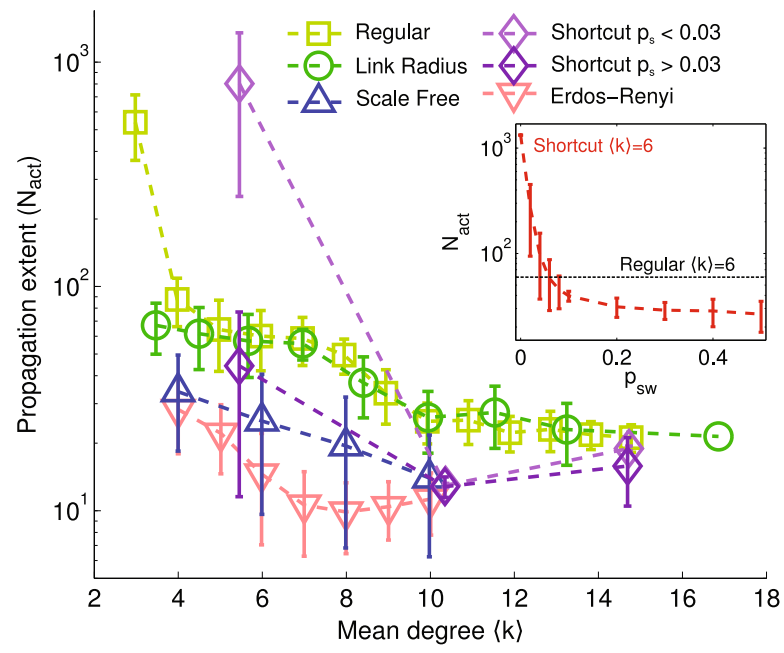

\subsubsection{Stimulation}

All the experiments are conducted using the same stimulation procedure: we stimulate one astrocyte in the center of the network (to avoid boundary effects on spatial networks) by coupling it during a stimulation time $t_{\text {stim }}$ via GJC to a virtual astrocyte whose $\mathrm{IP}_{3}$ cell concentration is kept constant at $\mathrm{IP}_{3}^{\text {bias }}$. All simulations are carried on during $t_{\text {sim }}=200 \mathrm{~s}$, giving enough time for a calcium wave to fully propagate to its maximum extent. The total number of activated cells during the simulation was found to be independent of $t_{\text {stim }}$ and $\mathrm{IP}_{3}^{\text {bias }}$ for $t_{\text {stim }} \geq 100 \mathrm{~s}$ and $\mathrm{IP}_{3}^{\text {bias }} \geq 2 \mu \mathrm{M}$, so we fixed during all simulations $t_{\text {stim }}=t_{\text {sim }}$ and $\mathrm{IP}_{3}^{\text {bias }}=2 \mu \mathrm{M}$.

\subsection{Results}

In order to distinguish topological effects from the effects introduced by GJC or biophysical parameters, we first carried out series of simulations with fixed GJC parameters $\left(F=2 \mu \mathrm{M} \mathrm{s}^{-1}\right.$ and $\left.\mathrm{IP}_{3}^{t h r}=0.3 \mu \mathrm{M}\right)$ while varying only the network topology.

\subsubsection{Topological Influences}

To ease comparison with experimental studies, we quantified the extent of wave propagation by measuring the total number of activated cells during the simulation, $N_{a c t}$, where a cell is considered activated at time $t$ if the amount of cytoplasmic calcium it contains is larger than $0.7 \mu \mathrm{M}$. Fig. 56.2 presents the variations of $N_{\text {act }}$ when the mean degree $\langle k\rangle$ varies; whatever the network model, the higher $\langle k\rangle$, the lower $N_{\text {act }}$. This is a first surprising result of our simulations: whatever the network type, 
Fig. 56.3 Relations between propagation extent $N_{a c t}$ and the mean-shortest path $L$ for each network model; error bars plot standard deviation in log scale. All models display the same qualitative behavior. Inset (1) represents a small example of propagation in an network strongly influenced by space while inset (2) uses a network weakly influenced by space. Green cells are activated cells that form the wave front; the wave is propagating from the bottom left corner to the top right one

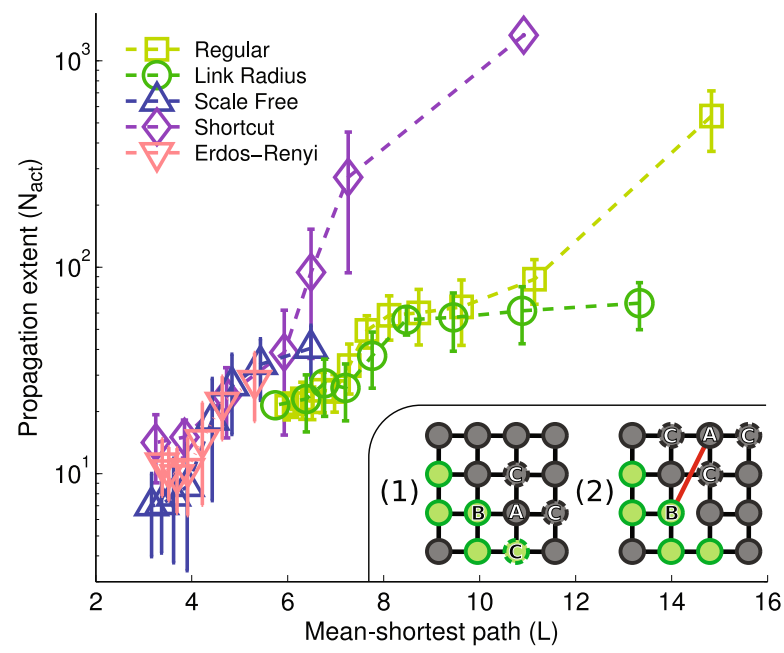

wave propagation is generically improved for networks with small mean degree. This result is however coherent with an intuitive understanding of the way $\mathrm{IP}_{3}$ diffuses in the network: in order to activate the astrocyte it arrives in (say astrocyte $A$ ), transferred $\mathrm{IP}_{3}$ must overcome a threshold value; therefore if $A$ is connected to a lot of inactive neighbors, most of this $\mathrm{IP}_{3}$ influx will leak to these inactive neighbors before it can activate $A$. Moreover, if it becomes activated, $A$ will give less $\mathrm{IP}_{3}$ to each of its unactive neighbors if it has a lot of unactive neighbors. Consequently, high mean-degree tends to impair calcium wave propagation.

However, for a given value of $\langle k\rangle, N_{a c t}$ can be down to 6-folds smaller in ErdősRényi networks than in regular or link radius networks. Therefore, the topology class still has an important effect on the propagation and $\langle k\rangle$ cannot alone explain the observed differences. Indeed, as we can see from the inset of Fig. 56.2, the shortcut network model goes from very large (spanning almost all the network) to very small extents, as shortcuts are more frequent while its mean degree is conserved. This behavior could hence be linked to the influence of space on topology: when links are restricted to a spatial neighborhood, the propagation extent is greater than when long distance links (or shortcuts) are added. Additionally, heavy space limitation also prevents the existence of highly connected nodes, influencing the mean degree $\langle k\rangle$. Furthermore, experimental findings indicate that real astrocyte networks are connected via a distance-controlled connectivity, i.e. large distance connexions are not likely (see [8, 9]).

This influence of space on topology can be quantified by the mean-shortest path $L$ of our networks as heavy space limitation yields high $L$ values and the addition of shortcuts reduces it.

Fig. 56.3 presents the relation between the propagation extent, $N_{a c t}$ and meanshortest path $L$. Again, all network models follow the same qualitative behavior: the larger the mean-shortest path, the better the propagation. This is a second surprising finding since it implies that, contrary to the intuition that shortcuts improve signal 
propagation in networks, the addition of shortcuts, decreasing L, actually prevents long range propagation in our model of astrocytic networks. But, just like for the conditions on $\langle k\rangle$ values, this relation between $L$ and the extent of propagation can be seen as the influence of space on networks.

Indeed, reliable activation of an astrocyte can be ensured if several of its neighbors are simultaneously active (its $\mathrm{IP}_{3}$ influx is then high enough to trigger a calcium spike) and heavy space constraints on topology is a way to ensure this. The rationale behind this affirmation is exposed by the two insets of Fig. 56.3; inset (1) presents a case in which space restriction is strong while inset (2) presents a case in which it is a bit relaxed, because of a "long distance" link (in red). The wave is propagating from the bottom left corner of the networks to the top right corner. An unactivated cell A is linked to an activated cell B in the "wavefront" (green cells); when space influence is high (inset (1)), cell A is spatially close to the wave front, its neighbors (marked C) are hence likely to be activated. Conversely, when space influence is low (inset (2)), the neighbors of A (marked C) are less likely to be in the wavefront because A can be spatially distant from the wavefront. Consequently, in our example, cell A is more likely to get activated when space influence is high (inset (1)) because it has two activated neighbors while it only has one when space influence is low (inset (2)).

However, this space influence cannot be captured by the mean clustering coefficient $\langle C\rangle$ of our networks. Indeed, as it is shown in the inset of Fig. 56.2, the propagation extent in 3D lattices $\left(\langle k\rangle=6, p_{s}=0\right.$ and $\left.\langle C\rangle=0\right)$ is larger than in regular networks $(\langle k\rangle=6$ and $\langle C\rangle=0.27 \pm 0.1)$. This particular behavior on lattices (also visible on Fig. 56.3) can be explained by considering the set of nodes that are within a given topological distance $l$ from a reference node (i.e. the shell $l$ of this reference node). Lattices have no links inside each shell; all the links are between shells. Hence a node in the shell $l+1$ will have a mean value of $\langle k\rangle / 2$ links coming from the shell $l$. In other network models, there are intra-shell links so the number of links between shell $l$ and shell $l+1$ is comparatively lower and activation is less easily transmited. This will not however be detailed here and is left to a future publication.

Therefore, according to our simulations, calcium wave propagation is expected to be favored in networks with large mean-shortest path, small mean degree and/or strong preference to connect only to nearest neighbors. In vivo, large regenerative waves spanning all the observable cells are sometimes (though rarely) observed (cf. [14]). Our results indicate that this could be linked to a transient decrease of gapjunction coupling levels, effectively leading to a reduction of the network mean degree, or to a restriction of gap-junction coupling to a few neighbors.

\subsubsection{Biophysical Influences}

In order to determine the influence of biophysical and GJC properties on propagation, we now fix the topology to the arguably more biologically realistic one, 
Fig. 56.4 Relations between $\chi$ and $k$ for different values of IP ${ }_{3}^{\text {thresh }}$; error bars plot the standard deviation. The inset represents the simple architecture that we used in order to determine these values

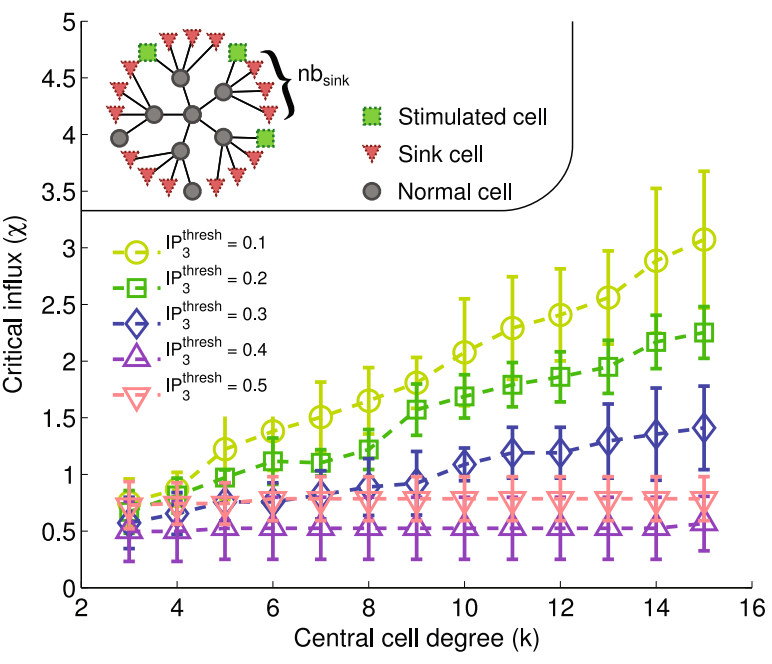

the link radius model, as links are expected to be restricted to nearest neighbors. We moreover fix the mean degree value to a realistic biological value of $k \approx 12$ (cf. [8]). We next studied the influence of the GJC parameters ( $F$ and $\mathrm{IP}_{3}^{\text {thresh }}$ ). The extent of propagation was not significantly affected by $F$ but was found to depend strongly on $\mathrm{IP}_{3}^{\text {thresh }}$, the minimum $\mathrm{IP}_{3}$ gradient above which $\mathrm{IP}_{3}$ is significantly transferred between the cells via GJC: the higher this parameter, the more individual astrocytes can store $\mathrm{IP}_{3}$ before diffusing it to neighboring unactivated astrocytes. Consequently, the propagation range $N_{\text {act }}$ was low (less than 50 cells) up to $\mathrm{IP}_{3}^{\text {thresh }}=0.3 \mu \mathrm{M}$ and for $\mathrm{IP}_{3}^{\text {thresh }} \geq 0.6 \mu \mathrm{M}$. However, for a restricted range of values $0.4 \leq \mathrm{IP}_{3}^{\text {thresh }} \leq 0.5 \mu \mathrm{M}$, propagation was strongly enhanced, reaching $N_{a c t} \sim 300$ activated astrocytes (compared to the $\sim 30$ activated astrocytes in previous simulations for the same topology).

In order to understand the strong effects of $\mathrm{IP}_{3}^{\text {thresh }}$ on the propagation extent, we conducted a systematic analysis of the activation conditions in the small network displayed at the top of Fig. 56.4. This network is made of one central cell, linked to $k$ neighbors, that are in turn each linked to one "normal" (stimulable) cell and $n b_{\text {sinks }}$ "sink" cells, that are artificially maintained in non activated state (red triangles on the figure). For each test, we chose a number of $n b_{\text {stim }}$ "normal" cells, among the $k$ ones that can be stimulated, and stimulate them (green squares on the figure). We then run the simulation and observe whether this number $n b_{\text {stim }}$ of stimulated cells is enough to activate the central cell. This simple network was designed so as to test the hypothesis that we formulated hitherto in order to explain how wave propagate in our networks: an activated cell (here the neighbors of the central cell) will more easily activate its neighbors (here we focus on the central cell) if it has few unactivated neighbors (the sink cells) to which $\mathrm{IP}_{3}$ can leak.

For fixed values of GJC parameters, $k$ and $n b_{\text {sinks }}$, we found that the central cell became activated only if $n b_{\text {stim }}$ overcomes a threshold $n b_{\text {stim }}^{\text {crit }}$. In stimulated branches of our network, when the cell connected to the central cell becomes activated, it 
passes a given total $\mathrm{IP}_{3}$ outflux $Q$ to its $n b_{\text {sink }}$ neighbor sinks and to the central cell (there is no flux to the stimulated cell as its $\mathrm{IP}_{3}$ level is already high). The partial flux received by the central cell from one of the $k$ branches is then $Q /\left(n b_{\text {sink }}+1\right)$ and the total flux received by the central cell is $n b_{\text {stim }} \times Q /\left(n b_{\text {sink }}+1\right)$. We define the normalized critical total influx needed in order to activate the central cell as:

$$
\chi=\frac{n b_{\text {stim }}^{\text {crit }}}{n b_{\text {sink }}+1}
$$

Figure 56.4 presents the values of $\chi$ as $k$ changes and for several values of $\mathrm{IP}_{3}^{\text {thresh }}$. It is apparent from this figure that the $\mathrm{IP}_{3}^{\text {thresh }}$ range for which we witnessed high propagation above $\left(\mathrm{IP}_{3}^{\text {thresh }} \in[0.4,0.5]\right)$ are remarkable here as well, since the values of the threshold $\chi$ do not depend on $k$ in this range of $\mathrm{IP}_{3}^{\text {thresh }}$ values. This means that, for these $\mathrm{IP}_{3}^{\text {thresh }}$ values, the central cell gets activated by a constant number of activating cells (a constant total influx $\chi$ ), regardless of its degree; $\mathrm{IP}_{3}$ leaking from the central cell does not prevent its activation. Conversely, for low values of $\mathrm{IP}_{3}^{\text {thresh }}$, the total number of activating cells needed in order to activate the central cell increases linearly with the number of its neighbors. This behavior is not, however, to be confused with a standard threshold model of propagation (cf. [3, 5, 21]) where a node gets activated if a fixed fraction of its neighbors are activated; in our case, one has also to take into account the neighborhood of the activated nodes: a given node is less activating if its own neighbors are unactivated (because $\mathrm{IP}_{3}$ will leak to them). Hence, in the specific case of calcium wave propagation in astrocyte networks, one needs to take into consideration the two-hop neighborhood of a node in order to determine whether it will get activated. Interestingly, one can define a generalized version of a threshold model that accounts for these two-hops neighborhood effects; presenting such a generalized model is beyond the scope of the present paper. We just note here that its behavior was found similar to those presented in Figs. 56.2 and 56.3.

Taken together, these results explain why the propagation extent $N_{a c t}$ depended on $\langle k\rangle$ in the above results. Indeed, all the above results have been obtained using $\mathrm{IP}_{3}^{\text {thresh }}=0.3$ for which $\chi$ increases with $k$. In this case, the influx threshold is increasingly high, rendering the propagation increasingly difficult, with increasing mean degrees. These results also show that the influence of topology on propagation is itself regulated by biophysical parameters (here GJC parameters); the resulting propagation is a complex interplay between these parameters and the network topology.

\subsection{Conclusion}

A major point in the results showed above is that we could obtain various extents of wave propagation by sole variations of the network topology; more precisely, two macroscopic measures on the network topology, its mean degree $\langle k\rangle$ and its mean-shortest path $L$ were enough to determine the extent of propagation. 
Table 56.1 Different types of waves can be reproduced by varying only the network topology

\begin{tabular}{llll}
\hline Wave type [exper. ref.] & $N_{a c t}$ & $\langle k\rangle$ & $L$ \\
\hline Locally synchronized [19] & $\sim 10$ & $\sim 8$ & $\sim 4$ \\
Spatially restricted [4] & $\sim 40$ & $\sim 7$ & $\sim 8$ \\
Regenerative [14, 20] & $\sim 500$ & $\sim 3$ & $\sim 14$ \\
\hline
\end{tabular}

Table 56.1 presents the three major types of waves reported in experimental articles with their respective extent. In our model, each type of wave can be reproduced using different values of $\langle k\rangle$ and $L$. Very small extents (locally synchronized event) can be obtained with relatively high degree networks and short mean-shortest path while regenerative waves (propagating to most of the cells) are triggered on small degree networks with high mean-shortest path. Therefore, large scale propagation is achieved when astrocytes are linked to nearest neighbors while linking to more (or more distant) astrocytes decreased propagation extent. Hence, while differences in propagation extent in the biological literature are usually explained by differences in biophysical parameters or in signaling pathways, we showed here that, in our model, topology alone was enough to explain these differences. As it is known that the structure of astrocytic networks varies throughout the brain and could even be shaped by neuronal activity [9], the influence of topology on wave propagation in vivo may be a fruitful hypothesis in order to understand the bidirectional relations between neurons and astrocytes at a network level.

For instance, our results could be evoked to formulate an hypothesis concerning the observation that regenerative waves usually occur in cultured astrocytes networks, where space has a very restrictive influence on the links, as these in vitro networks are constrained to a 2D surface. This would strongly reduce the number of close neighbors thus, according to our model, favor long-range propagation .

\section{References}

1. Barthélemy M (2011) Spatial networks. Phys Rep 499(1-101):2010

2. Bushong EA, Martone ME, Jones YZ, Ellisman MH (2002) Protoplasmic astrocytes in ca1 stratum radiatum occupy separate anatomical domains. J Neurosci 22(1):183-192

3. Centola D, Eguíluz VM, Macy MW (2007) Cascade dynamics of complex propagation. Physica A 374(1):449-456

4. Charles A (1998) Intercellular calcium waves in glia. GLIA 24(1):39-49

5. Dodds PS, Watts DJ (2004) Universal behavior in a generalized model of contagion. Phys Rev Lett 92(21):218701

6. Dokukina I, Gracheva M, Grachev E, Gunton J (2008) Role of network connectivity in intercellular calcium signaling. Physica D 237(6):745-754

7. Edwards JR, Gibson WG (2010) A model for ca2+ waves in networks of glial cells incorporating both intercellular and extracellular communication pathways. J Theor Biol 263(1):45-58

8. Giaume C (2010) Astroglial wiring is adding complexity to neuroglial networking. Front Neuroenerg 2:129

9. Giaume C, Koulakoff A, Roux L, Holcman D, Rouach N (2010) Astroglial networks: a step further in neuroglial and gliovascular interactions. Nat Rev Neurosci 11(2):87-99 
10. Goldberg M, Pittà MD, Volman V, Berry H, Ben-Jacob E (2010) Nonlinear gap junctions enable long-distance propagation of pulsating calcium waves in astrocyte networks. PLoS Comput Biol 6(8):e1000909

11. Höfer T, Venance L, Giaume C (2002) Control and plasticity of intercellular calcium waves in astrocytes: a modeling approach. J Neurosci 22(12):4850-4859

12. Kang M, Othmer HG (2009) Spatiotemporal characteristics of calcium dynamics in astrocytes. Chaos 19(3):037116

13. Kazantsev VB (2009) Spontaneous calcium signals induced by gap junctions in a network model of astrocytes. Phys Rev E, Stat Nonlinear Soft Matter Phys 79(1):010901

14. Kuga N, Sasaki T, Takahara Y, Matsuki N, Ikegaya Y (2011) Large-scale calcium waves traveling through astrocytic networks in vivo. J Neurosci 31(7):2607-2614

15. Ogata K, Kosaka T (2002) Structural and quantitative analysis of astrocytes in the mouse hippocampus. Neuroscience 113(1):221-233

16. Perea G, Araque A (2010) Glia modulates synaptic transmission. Brains Res Rev 63(1-2):93102

17. Pittà MD, Volman V, Levine H, Pioggia G, Rossi DD, Ben-Jacob E (2008) Coexistence of amplitude and frequency modulations in intracellular calcium dynamics. Phys Rev E, Stat Nonlinear Soft Matter Phys 77(3):030903

18. Roux L, Benchenane K, Rothstein JD, Bonvento G, Giaume C (2011) Plasticity of astroglial networks in olfactory glomeruli. Proc Natl Acad Sci USA. doi:10.1073/pnas.1107386108

19. Sasaki T, Kuga N, Namiki S, Matsuki N, Ikegaya Y (2011) Locally synchronized astrocytes. Cereb Cortex. doi:10.1093/cercor/bhq256

20. Scemes E, Giaume C (2006) Astrocyte calcium waves: what they are and what they do. GLIA 54(7):716-725

21. Watts DJ (2002) A simple model of global cascades on random networks. Proc Natl Acad Sci USA 99(9):5766-5771 